http://jmscr.igmpublication.org/home/

ISSN (e)-2347-176x ISSN (p) 2455-0450

crossref DOI: https://dx.doi.org/10.18535/jmscr/v7i8.164

Journal Of Medical Science And Clinical Research

\title{
Chemoradiotherapy of Locally-Advanced Anal Canal Squamous Cell Carcinoma: A Brief Review of Outcomes of Randomized Clinical Trials
}

Authors

\author{
Erkan Topkan, MD ${ }^{1}$, Huseyin Mertsoylu, MD $^{2}$, Ahmet Kucuk, MD $^{3}$
}

${ }^{1}$ Baskent University Medical Faculty, Department of Radiation Oncology, Adana, Turkey

${ }^{2}$ Baskent University Medical Faculty, Department of Medical Oncology, Adana, Turkey

${ }^{3}$ Mersin City Hospital, Radiation Oncology Clinics, Mersin, Turkey

*Corresponding Author

Erkan Topkan, MD

Baskent University Medical Faculty, Department of Radiation Oncology, 01120, Adana, TURKEY

\begin{abstract}
First-line treatment of locally advanced anal canal squamous cell carcinomas (AC-SCC) has evolved from the abdominoperineal resection resulting in permanent colostomies to definitive concurrent chemoradiotherapy, with surgery reserved for salvage. Publication of small but practice-changing chemoradiotherapy studies in the second half of the 1970s prompted the conduction of large phase III randomized controlled trials consequences of which increased our ability to deliver systemic chemotherapy more effectively and reduce treatment-related toxicity rates by utilizing more sophisticated radiotherapy techniques. This article aims to review the design and outcomes of currently accessible phase III randomized controlled chemoradiotherapy trials of AC-SCC.
\end{abstract}

\section{Introduction}

Anal canal squamous cell carcinoma (AC-SCC) is a relatively rare tumor which constitutes only $2 \%$ of all gastrointestinal tumors ${ }^{(1,2)}$. However, the incidence of AC-SCC has been increasing over the past three 30 years, which is assumed to be associated with increased exposure to the human papillomavirus and human immunodeficiency virus infections, for the most part, due to changes in sexual practices ${ }^{(2)}$.

Abdominoperineal resection (APR) with resultant permanent colostomy was the standard of care for all stages of $\mathrm{AC}-\mathrm{SCC}^{(3)}$ until the publication of seminal work by Nigro $1974^{(4)}$. In this small yet admirable study, the authors exhibited the superiority of neoadjuvant concurrent chemoradiotherapy (C-CRT) over surgery alone with utilizing 30 Gy (15 fractions) radiotherapy (RT) and concurrently administered 5-fluorouracil (5-FU) and mitomycin-C (MMC). Opening another treatment window for AC-SCC, the authors reported that the pathologic complete response (CR) was achieved in $80 \%$ of patients following this regimen. Albeit initially the practice-changing Nigro's C-CRT protocol was developed as a neoadjuvant therapy preceding surgical resection, the exhibition that $80 \%$ of patients were rendered free of cancer by C-CRT on clinical and histopathological examinations prompted the conduction of many prospective 
studies to validate these results and to investigate the C-CRT as the primary definitive treatment option for such patients ${ }^{(5)}$.

The remaining part of this article will briefly discuss the outcomes of the accessible large-scale phase III randomized controlled trials (RCTs) of C-CRT in SC-ACC patients, with specific emphasis on the potential factors those may have altered the presented results.

\section{Randomized Controlled Trials}

After the publication of the promising results of the small C-CRT studies, large RCT's were designed to validate definitive C-CRT as the primary treatment for AC-SCC. Moreover, these RCTs sought for the most effective and less toxic chemotherapeutics, the potential benefits of the induction and maintenance chemotherapy, escalated doses of RT, and optimal timing of the response assessment. Regardless of the differences among the secondary objectives of the RCTs, the common ultimate objective of these endeavors incorporated the provision of maximal colostomyfree survival with minimal acute and/or late treatment-related toxic event rates. The synopsis of the results of the accessible RCTs is as delineated in Table 1.

\section{Anal Canal Trial I}

The United Kingdom Coordinating Committee on Cancer Research (UKCCR) Anal Canal Trial I (ACT I) was a multicenter trial conducted to compare C-CRT against RT alone (6). In this benchmark study, 585 patients with AC-SCC were randomized to receive initially either 45 Gy pelvic RT (20-25 fractions) over 4-5 weeks $(n=290)$ or the same RT regime concurrent with continuously infused 5 -FU $\left(1000 \mathrm{mg} / \mathrm{m}^{2}\right.$ for 4 days or 750 $\mathrm{mg} / \mathrm{m}^{2}$ for 5 days) during the first and the last weeks of RT and MMC $\left(12 \mathrm{mg} / \mathrm{m}^{2}\right)$ on the first day of RT $(n=295)$. The anteroposteriorposteroanterior opposed RT fields were designed to involve anus, perineum, lower pelvic lymph nodes (LNs), with RT for inguinal LNs being optional. Of the 585 patients, $51 \%$ had clinical $\mathrm{T}_{3}$ disease and $20 \%$ LN involvement, respectively. Clinical response was assessed 6 weeks after the completion of RT or C-CRT. Poor- $(<50 \%$ response) or non-responders were referred to immediate salvage surgery, while good responders (>50\%) received boost RT (15 Gy in 6 fractions) or brachytherapy (25 Gy in 2.5 days) to primary site. The primary endpoint was the clinically confirmed local failure at post-treatment 6 weeks. Secondary endpoints included the overall- (OS) and cause-specific survival (CSS), respectively.

Despite the clinical response rates at first clinical examination were similar (92\%), yet local failures were significantly more common in the RT than the C-CRT arm (59\% vs. $36 \%$; $\mathrm{P}<0.0001)$ after a median follow-up of 42 months. Albeit the OS was not improved $(\mathrm{P}=0.25)$, the CSS was significantly superior in the C-CRT arm $(\mathrm{P}=$ 0.02), which was gained at the cost of significantly higher acute $(\mathrm{P}=0.003)$ but not late toxicity rates. Based on these favorable results, the authors recommended the C-CRT as the new standard curative treatment for most AC-SCC patients with surgery being reserved for recurrences.

In the more recent update of ACT I published in 2010 , the long term results with a median followup of 13 years revealed that the 5-year local control (68 vs. $43 \%$; $\mathrm{P}<0.001)$ and CSS $(70 \%$ vs. $58 \%$; $\mathrm{P}=0.004)$ rates favored the $\mathrm{C}-\mathrm{CRT}$ over RT alone arm which were maintained at 10 years, as well (7). The colostomy-free survival (CFS), not reported in the initial report, also favored the $\mathrm{C}$ CRT arm (47 vs. 37\%; $\mathrm{P}=0.004$ ). These gains were shown to be brought with no significant differences in late toxicity rates of the two treatment arms. However, likewise the initial report, these favorable outcomes could not translate into a notable OS gain at 5 years $(58 \%$ vs. $53 \%$; $=0.12$ ), which may be associated with the more frequent utilization of the salvage surgery option in the RT alone arm.

In summary, setting the 45 Gy pelvic RT and concurrent 5-FU/MMC as the new standard of care for the most AC-SCC patients, the UKCCR ACT I trial demonstrated the superiority of the CCRT over RT alone for such patients in terms of 
improved locoregional control (LRC), CFS, and CSS rates.

\section{European Organization for the Research and Treatment of Cancer 22861}

European Organization for the Research and Treatment of Cancer 22861 trial (EORTC 22861) was a smaller phase $3 \mathrm{RCT}$ with a similar study design to ACT I, that randomized 110 AC-SCC into RT alone and C-CRT arms ${ }^{(8)}$. Randomized patients had $\mathrm{T}_{3-4} \mathrm{~N}_{0-3}$ or $\mathrm{T}_{1-2} \mathrm{~N}_{1-3}$ disease stages who received a total of 45 Gy (1.8 Gy per fraction) utilizing a 3- or 4-field pelvic RT technique. Patients were assessed for clinical response 6 weeks after the last day of the RT. If not contraindicated, surgical resection as a part of the primary treatment was performed in patients who had no response or judged to have a residual palpable disease. Differing from the ACT I, the boost RT dose was determined according to the extent of response in this study: $15 \mathrm{~Gy}$ for complete responders and $20 \mathrm{~Gy}$ for partial responders, respectively. In the C-CRT arm, the chemotherapy consisted of $750 \mathrm{mg} / \mathrm{m}^{2}$ daily $5-\mathrm{FU}$ as a continuous infusion administered on days 1-5 and 29-33, and bolus MMC $15 \mathrm{mg} / \mathrm{m}^{2}$ on day 1 of RT.

The patients in the C-CRT arm exhibited an essentially higher $\mathrm{CR}$ rate $(80 \%)$ than their RT alone arm (54\%) companions, which translated to significantly enhanced 5-year LRC (68\% vs. 50\%; $\mathrm{P}=0.02$ ) and CFS (72 vs. $40 \% ; \mathrm{P}=0.002$ ) rates. In spite of a pattern trend for higher anal ulceration rate in the C-CRT arm, no noteworthy distinction was found when serious late symptoms were considered. Likewise the ACT I, these outstanding gains did not lead to any OS advantage, being $56 \%$ at 5 -year in both arms.

In summary, the results of the EORTC 22861 trial affirmed the outcomes of the larger ACT I trial, and these two trials set the pelvic RT plus concurrent 5-FU/MMC combination as the new standard treatment for the AC-SCC patients.

\section{The United States Intergroup Trial}

The United States Intergroup Trial [Radiation Therapy Oncology Group (RTOG) 87-04/Eastern
Cooperative Oncology Group (ECOG) 2289] represents for the unique phase $3 \mathrm{RCT}$ that aimed to investigate whether the addition of MMC to 5FU was vital for better results in AC-SCC compared to 5-FU alone ${ }^{(9)}$. This milestone investigation was conducted mainly to address the hoped reductions in MMC-induced severe hematologic toxicities. A sum of 310 patients was randomized to one of the RT plus 5-FU or RT plus 5-FU/MMC combination arms. Of 291 assessable patients, 145 received 45-50.4 Gy of pelvic RT plus 5 -FU at $1,000 \mathrm{mg} / \mathrm{m}^{2} / \mathrm{d}$ for 4 days, and 146 received RT, $5-\mathrm{FU}$, and MMC $\left(10 \mathrm{mg} / \mathrm{m}^{2}\right.$ per dose for two doses). The study design allowed to enroll patients with any $\mathrm{T}$ and $\mathrm{N}$ stages: $17 \%$ was $\mathrm{N}+$ and $85 \%$ had $\mathrm{T}_{2-4}$ primaries. Shrinking pelvic RT technique was utilized and all patients received 45 Gy pelvic RT (1.8 Gy per fraction) with permission for an additional $5.4 \mathrm{~Gy}$ boost for the residual primary or nodal disease. Patients with residual tumors on posttreatment sixth-week week biopsy underwent a salvage regimen that consisted of an additional 9 Gy RT to the residual primary and/or palpable inguinal nodes plus 5FU/cisplatin combination. Cisplatin was allowed to be replaced with MMC in patients with decreased kidney functions.

Post treatment biopsies were positive in $15 \%$ of patients in the 5-FU and 7.7\% in the 5-FU/MMC arms $(\mathrm{P}=0.135)$, respectively. At 4 years, colostomy rates were lower (9 vs. $22 \%$; $\mathrm{P}=0.002$ ), while CFS (71\% v 59\%; $\mathrm{P}=0.014)$ and diseasefree survival (DFS) rates were higher $(73 \% \mathrm{v}$ $51 \% ; \mathrm{P}=0.0003$ ) in the 5-FU/MMC arm. Although the addition of MMC conferred better clinical outcomes in terms of reduced locoregional failure (LRF) and colostomy rates, these did not bring a remarkable OS advantage at 4 years.

In summary, despite no OS significant gain was observed, the reduced colostomy and LRF rates, and superior DFS served with MMC affirmed the MMC as an indispensable component of chemotherapy and set the RT plus 5FU/MMC combination as the standard C-CRT regimen for AC-SCC patients. 


\section{Radiation Therapy Oncology Group 98-11 Trial}

The RTOG 98-11 is one of three RCTs investigating the likelihood for supplanting the possibility for replacement of MMC with another chemotherapeutic agent to decrease severe hematologic toxicities and improve the accessible clinical outcomes ${ }^{(10)}$. In this large phase III RCT, $682 \mathrm{~T}_{2-4}$ and any $\mathrm{N}$ patients with AC-SCC were randomly assigned to one of the standard RT plus concurrent 5-FU/MMC and investigational induction 5-FU/cisplatin combination pursued by the RT plus concurrent 5-FU/cisplatin treatment arms. Of the 682 patients, 35\%, 26\% , and $27 \%$ had $\mathrm{T}_{3-4}$ primaries, $\mathrm{N}+$ disease, and $>5 \mathrm{~cm}$ tumors, respectively. Multiple fields pelvic RT technique was utilized and a total dose of 45 Gy (1.8 Gy per fraction) administered to all patients with an additional 10 to 14 Gy (2 Gy per fraction) boost being delivered to the primary tumor and $\mathrm{N}+$ sites. This RCT differed from the ACT 1, EORTC 2861, and RTOG 87-04 by its study design which did not mandate a planned break between the initial pelvic RT and residual tumor boost phases.

At a median follow-up of 2.51 years, despite the fact that initial results fundamentally favored the MMC arm over the cisplatin arm considering 3year colostomy rates (10 vs. $16 \%$; $\mathrm{P}=0.02$ ), yet there were no noteworthy differences between the two arms with respect to the 3- year DFS (67 vs. $61 \% ; \mathrm{P}=0.17$ ) and $\mathrm{OS}(84$ vs. $76 \% ; \mathrm{P}=0.10)$ rates, individually. Albeit the acute and late toxicity outcomes were in general similar in both groups, yet grade 3-4 acute hematologic toxicity rates were significantly higher in the MMC than the cisplatin arm (61 vs. $42 \%$; $\mathrm{P}<0.001)$. In the longterm update reported by Gunderson et $\mathrm{al}^{(11)}$, the cisplatin arm appeared to prove inferior to standard MMC arm in terms of 5-year CFS (65\% vs. $72 \% ; \mathrm{P}=0.05$ ), DFS (58 vs. $68 \% ; \mathrm{P}=0.006$ ), OS (71 vs. $78 \% ; \mathrm{P}=0.026$ ), and cumulative colostomy need $(19 \%$ vs. $10 \% ; \quad \mathrm{P}=0.02)$, separately..

In the RTOG 98-11 trial, induction chemotherapy may have decreased the tumor response to the radio sensitizing effects of cisplatin, and furthermore, the delayed C-CRT in the induction chemotherapy may have induced the wellperceived accelerated repopulation phenomenon, which altogether may have adversely altered the outcomes in the induction arm. Whatever the reason was, the outcomes of RTOG 98-11 confirmed the essential role of MMC and settled the immediate C-CRT with 5-FU/MMC as the standard of consideration for the suitable AC-SCC patients.

\section{Anal Cancer Trial II}

The RTOG 98-11 compared the 5-FU/MMC with 5-FU/cisplatin and exhibited that the replacement of MMC with cisplatin resulted in inferior clinical outcomes. However, as the RTOG 98-11 design brought the likelihood of loss of the radiosensitizing actions of cisplatin together with the consequences of the ominous radiobiologic impact of deferred C-CRT, this study was condemned to not respond to the inquiry whether the hematologically severe toxic MMC might be securely supplanted by cisplatin. Additionally, the use of the induction chemotherapy led to indirect comparisons between the two chemotherapy regimens rather than head to head direct comparisons.

In this regard, the Anal Cancer Trial II (ACT II) was a large phase III RCT directly investigating the influence of replacement of MMC with cisplatin on patients' outcomes ${ }^{(12)}$. Further, this $2 \times 2$ randomized trial addressed the impact of addition of maintenance chemotherapy to standard C-CRT in 940 AC-SCC patients from 59 UK centers: patients were randomly assigned to one of four groups, to receive either MMC $\left(12 \mathrm{mg} / \mathrm{m}^{2}\right.$ on day 1) or cisplatin $\left(60 \mathrm{mg} / \mathrm{m}^{2}\right.$ on days 1 and 29), with 5 -FU $\left(1000 \mathrm{mg} / \mathrm{m}^{2}\right.$ per day on days $1-4$ and 29-32) and pelvic RT (50.4 Gy in 28 fractions); with/without two courses of maintenance 5FU/cisplatin at weeks 11 and 14. Pelvic RT consisted of anteroposterior-posteroanterior fields with a field reduction at 30.6 Gy and carried out to a total dose of $50.4 \mathrm{~Gy}$. The treatment response was assessed three times at 11, 18, and 26 weeks 
after the last day of RT. The primary objectives were CR at 26 weeks and acute toxicity rates (for C-CRT), and PFS (for maintenance). The CR rates at 26 weeks examinations were reported to be not different between the MMC and cisplatin arms. (90.5\% vs. $89.6 \%$; $\mathrm{P}=0.64$ ), respectively. Only $44 \%$ of the maintenance cohort could receive the prescribed full-dose treatment. The 5-year PFS was similar between the maintenance and no maintenance arms (74 vs. $73 \% ; \quad \mathrm{P}=0.70)$. Additionally, regardless of the maintenance status, there were no notable differences between the CFS and OS rates between the treatment groups. Other than the more frequent hematologic toxicities in the MMC arms, there were no significant differences between the acute and late toxicity outcomes between the randomization arms.

In the recently reported post hoc analysis of ACT II (13), the outcomes of the LN response was also provided in addition to the primary site, which was not available in the initial report. The authors noted that the CR rate was increased by $21 \%$ (64 vs. $85 \%$ ) between the assessment weeks 11 and 26. Additionally, the investigators examined the OS outcomes in regards to the CR rates achieved at weeks 11 and 26. Accordingly, complete responders at week 11 had a numerically superior but not significant 5-year OS rates compared to those patients with less than CR (85 vs. 75\%; $\mathrm{P}=0.38$ ). But interestingly, the 5-year OS appeared to favor complete responders at week 1126 in a statistically significant manner (87 vs. $48 \%$; $\mathrm{P}<0.001)$.

In summary, after the RTOG 98-11 which reported no benefit for induction chemotherapy, the largest of ever AC-SCC trial ACT II demonstrated that the maintenance chemotherapy had no role in the standard management of ACSCC. An additional important finding of the further post hoc analysis of ACT II was the discovery of the 26 weeks (delayed) response assessment with superior prognostic value compared to week 11 (earlier) examinations, which supports the notion proposing continuing tumor regression by time after the standard CCRT. The ACT II trial also suggested the utilization of 5FU/cisplatin in patients anticipated to be not able to tolerate the severe hematologic toxicity of 5FU/MMC, although not recommended for every AC-SCC patients.

\section{Action Clinique Coordonnées en Cancérologie Digestive-03}

The Action Clinique Coordonnées en Cancérologie Digestive-03 (ACCORD 03) was designed to determine whether dose escalation of the RT boost or 2 cycles of induction chemotherapy before C-CRT lead to an improvement in CFS rates ${ }^{(14)}$. The ACCORD 03 trial was also a $2 \times 2$ design RCT like the ACT II, but differing from the aforementioned $5 \mathrm{C}-\mathrm{CRT}$ trials the MMC was excluded in the chemotherapy convention. Patients with tumors $\geq 4 \mathrm{~cm}$, or $<4 \mathrm{~cm}$ and $\mathrm{N}_{1-3}$ were randomly assigned to one of 4 treatment arms: Arm 1: Two cycles of induction chemotherapy (5-FU $800 \mathrm{mg} / \mathrm{m} 2 /^{\mathrm{d}}$, days $1-4$ and 29- 32; and cisplatin $80 \mathrm{mg} / \mathrm{m}^{2}$, days 1 and 29), CCRT (45 Gy in 25 fractions, 5-FU/cisplatin, weeks 1 and 5), and standard-dose boost (SDB; 15 Gy); Arm 2:Two cycles of induction chemotherapy, CCRT, and high-dose boost (HDB; 20-25 Gy); Arm 3: C-CRT and SDB (reference arm); and Arm 4: C-CRT and HDB. The boost RT was administered after a 3 weeks break following the completion of the standard pelvic RT.

The intended C-CRT was completed by $94 \%$ to 98\% patients in the non-induction arms, while these rates decreased to $79 \%$ to $82 \%$ in the induction arms. At a median follow-up of 4.2 years, the 5-year CFS rates were $69.6 \%, 82.4 \%$, $77.1 \%$, and $72.7 \%$ in arms $1,2,3$, and 4 , respectively. Considering the $2 \times 2$ factorial analysis, the 5-year CFS was $76.5 \%$ versus $75.0 \%$ $(\mathrm{P}=0.37)$ in groups $\mathrm{A}$ and $\mathrm{B}$ versus $\mathrm{C}$ and $\mathrm{D}$, respectively (induction chemotherapy effect), and $73.7 \%$ versus $77.8 \%$ in groups $\mathrm{A}$ and $\mathrm{C}$ versus $\mathrm{B}$ and $\mathrm{D}$, respectively (RT-dose effect; $\mathrm{P}=0.067$ ).

In summary, the consequences of the ACCORD 03 trial demonstrated that neither the induction chemotherapy nor the escalated doses of RT boost 
had the option to improve the results of standard C-CRT, albeit the escalated dose RT had an effect near significance. However, again the 3 weeks break between the two phases of RT may have shadowed the small but significant effect of escalated dose RT. Therefore, based on these results, immediate $\mathrm{C}-\mathrm{CRT}$ retains its evidencebased standard role in the treatment of AC-SCC patients.

Table 1 Randomized controlled trials of concurrent chemoradiotherapy trials in anal canal squamous cell carcinoma

\begin{tabular}{|c|c|c|c|c|c|}
\hline Trial (Reference) & $\begin{array}{l}\text { Patients } \\
\text { (n) }\end{array}$ & Treatment Arms & LRC & $\overline{\mathrm{OS}}$ & CFS \\
\hline ACT-I $(6,7)$ & 585 & $\begin{array}{c}\mathrm{RT} \\
\mathrm{RT}+5-\mathrm{FU} / \mathrm{MMC}\end{array}$ & $\begin{array}{c}43 \%(5 \text { years }) \\
68 \%\end{array}$ & $\begin{array}{c}53 \%(5 \text { years }) \\
58 \%\end{array}$ & $\begin{array}{c}37 \% \text { (5 years) } \\
47 \%\end{array}$ \\
\hline EORTC 22861 (8) & 110 & $\begin{array}{c}\mathrm{RT} \\
\mathrm{RT}+5-\mathrm{FU} / \mathrm{MMC}\end{array}$ & $\begin{array}{c}50 \%(5 \text { years }) \\
68 \%\end{array}$ & $\begin{array}{c}56 \% \\
56 \%(5 \text { years })\end{array}$ & $\begin{array}{c}40 \% \text { (5 years) } \\
72 \%\end{array}$ \\
\hline RTOG 8704 (9) & 310 & $\begin{array}{c}\mathrm{RT}+5-\mathrm{FU} \\
\mathrm{RT}+5-\mathrm{FU} / \mathrm{MMC}\end{array}$ & $\begin{array}{c}66 \%(4 \text { years }) \\
84 \%\end{array}$ & $\begin{array}{c}67 \% \text { (4 years) } \\
76 \%\end{array}$ & $\begin{array}{c}59 \% \text { (4 years) } \\
71 \%\end{array}$ \\
\hline $\begin{array}{l}\text { RTOG } \\
(\mathbf{1 0 , 1 1 )}\end{array}$ & 682 & $\begin{array}{c}\mathrm{RT}+5-\mathrm{FU} / \mathrm{MMC} \\
\text { Induction 5-FU/Cisplatin + } \\
\mathrm{RT}+5-\mathrm{FU} / \text { Cisplatin }\end{array}$ & $\begin{array}{c}80 \%(5 \text { years }) \\
74 \%\end{array}$ & $\begin{array}{c}78 \% \text { (5 years) } \\
71 \%\end{array}$ & $\begin{array}{c}72 \%(5 \text { years }) \\
65 \%\end{array}$ \\
\hline ACT-II $(12,13)$ & 940 & $\begin{array}{c}\mathrm{RT}+5-\mathrm{FU} / \mathrm{MMC} \\
\mathrm{RT}+5-\mathrm{FU} / \text { Cisplatin }\end{array}$ & NA & $\begin{array}{c}79 \% \\
77 \% \text { (5 years) }\end{array}$ & $\begin{array}{c}68 \% \\
67 \% \text { (5 years) }\end{array}$ \\
\hline ACCORD-03 (14) & 307 & $\begin{array}{c}\mathrm{RT}+5-\mathrm{FU} / \text { Cisplatin } \\
\text { Induction 5-FU/Cisplatin + } \\
\mathrm{RT}+\text { 5-FU/Cisplatin }\end{array}$ & $\begin{array}{c}81 \% \text { (5 years) } \\
80 \%\end{array}$ & $\begin{array}{c}71 \%(5 \\
\text { years }) 74 \%\end{array}$ & $75 \%$ (5 years) $76 \%$ \\
\hline
\end{tabular}

Abbreviations: CFS: Colostomy-free survival; LRC: Locoregional control; OS: Overall survival; RT: Radiotherapy; 5-FU: 5 Fluorouracil; MMC: Mitomycin C; NA: Not available; ACT: Anal Canal Trial; EORTC: European Organization for the Research and Treatment of Cancer: RTOG:Radiation Therapy Oncology Group; ACCORD: Action Clinique Coordonnées en Cancérologie Digestive.

Table 2 Established prognostic factors for anal canal squamous cell carcinomas according to the end-points derived from the randomized controlled trials

\begin{tabular}{llllll}
\hline Factor & CFS & ACSS & DFS & LRFFS & OS \\
\hline Gender & - & Yes & - & Yes & Yes \\
Tumor ulceration & - & - & - & Yes & Yes \\
Histologic differentiation & - & - & - & - & Yes \\
Anemia & - & Yes & - & - & \\
Tumor stage & Yes & - & Yes & - & Yes \\
Nodal stage & - & Yes & - & Yes & Yes \\
\hline
\end{tabular}

Abbreviations: CFS: Colostomy-free survival; ACSS: Anal cancer specific survival;DFS Disease-free survival; LRFFS: Locoregional failure-free survival; OS: Overall survival

\section{Discussion}

Compared to 1970's standards, the management of the locally-advanced AC-SCC evolved from the debilitating abdominoperineal resection with a perpetual colostomy to sphincter preserving CCRT. The major finding of the accessible large phase III RCTs was the exhibition of the superiority of the concurrently administered RT plus FU/MMC over the RT alone conventions in terms of better response rates, LRC, CSF, and DFS rates. Nonetheless, aside from the RTOG 9811 , these benefits never translated into an OS advantage which might be related to the long survival times accomplished with standard C-CRT in an average AC-SCC. Additional RCTs were not able to show an alternative chemotherapy agent which may replace the MMC to overcome its hematologic toxicity. In this regard, intensitymodulated RT (IMRT) may demonstrate useful in reducing the acute toxicities including the severe acute hematologic toxic events with its more conformal dose distribution as proven by the RTOG 0529 trial $^{(15,16)}$. 
Induction chemotherapy and escalated doses of RT boost were also investigated, but unfortunately, both appeared to have no valuable consequences for the results. However, the result should be interpreted with caution as the splitcourse RT may have masked the presence of a moderate but significant effect of escalated dose $\mathrm{RT}$, as suggested by the results of the ACCORD 03 trial. This issue may merit further examination in the setting of concurrent simultaneous integrated boost IMRT.

Serving as a potential therapeutic target epidermal growth factor receptor (EGFR) is over expressed in about $90 \%$ of all AC-SCCs. In the Olivatto and associates' investigation assessing the utility of cetuximab, the authors administered cetuximab with cisplatin and 5-FU during C-CRT ${ }^{(17)}$. Even though the pathologic complete reaction of $95 \%$ was encouraging with a 3-year LRC rate of $64.2 \%$, yet as all of the 23 patients enrolled experienced various grade 3/4 toxicities (100\%), hence, this study was closed earlier due to safety concerns. In like manner, the UNICANCER ACCORD 16 phase II trial in which the same treatment protocol was utilized in 16 patients with AC-SCC was also prematurely closed as $88 \%$ of patients experienced severe toxicities ${ }^{(18)}$. In the more recent Eastern Cooperative Oncology Group-American College of Radiology Imaging Network Cancer Research Group Trial (E3205) trial, 61 immunocompetent AC-SCC patients received C-CRT including cisplatin, 5-FU plus eight once-weekly doses of concurrent cetuximab (19). This study was designed to detect at least a $50 \%$ reduction in 3-year LRF rate assuming a 35\% LRF rate from historical data. Unfortunately, again this regimen was found to be highly toxic with $32 \%$ and $5 \%$ grade 4 toxicity and treatmentrelated death rates. Therefore, further studies on EGFR inhibitors are required to decide their actual incentive in treatment of AC-SCCs. Furthermore, their combinations with the infrequently referred novel immunotherapies may likewise demonstrate valuable in the enhancement of the outcomes of these patients group, albeit no strong information exists on the utilization of immunotherapeutics in the setting of locally advanced AC-SCCs ${ }^{(20)}$. Although numerous clinical and molecular factors have been proposed to have prognostic impacts on various endpoints in past examinations, another contribution of the reported RCTs is the foundation of the prognostic factors in AC-SCC patients undergoing C-CRT. As summarized in Table 2, to date only six factors have been proven to demonstrate prognostic utility for AC-SCC patients. Accessibility of such factors may conceivably serve helpful in appropriate prognostic stratification of AC-SCC patients and their assignment into study arms with differential treatment intensities in future trials.

In conclusion, based on the accessible solid evidence, the current National Comprehensive Cancer Network and European Society for Medical Oncology guidelines recognizes and recommends the RT plus concurrent 5-FU/MMC combination as the standard of care for the locally-advanced AC-SCC patients, However, although cannot be recommended for all patients, based on the outcomes of the ACT II trial 5 FU/cisplatin may be preferred for patients anticipated to be not able to tolerate the severe hematologic toxicity of 5FU/MMC combination. As of now the EGFR inhibitors and immunotherapeutics remain investigational and ought to be utilized in the research settings.

\section{Research Support: None}

Conflicts of Interest: None to declare

Author contributions: All authors contributed equally

\section{References}

1. Nelson VM, Benson AB 3rd. Epidemiology of Anal Canal Cancer.. Surg Oncol Clin N Am. 2017;26(1):9-15.

2. Glynne-Jones R, Rao S. Treatment of the Primary Tumor in Anal Canal Cancers. Surg Oncol Clin N Am. 2017;26(1):73-90.

3. Sato H, Koh P, Bartolo DC. Management of anal canal carcinoma. Dis Colon Rectum 2005;48:1301-15. 
4. Nigro ND, Vaitkevicius VK, Considine B. Combined therapy for cancer of the anal canal: a preliminary report. Dis Colon Rectum 1974;17:354-6.

5. Leichman L, Nigro ND, Vaitkevicius VK. Cancer of the anal canal. Model for preoperative adjuvant combined modality therapy. Am J Med. 1985;78(2):211-5.

6. Northover J, Glynne-Jones R, Cunningham D, et al. Epidermoid anal cancer: results from the UKCCCR randomised trial of radiotherapy alone versus radiotherapy, 5-fluorouracil, and mitomycin. Lancet. 1996;348(9034):104954.

7. Northover J, Glynne-Jones R, SebagMontefiore D, et al. Chemoradiation for the treatment of epidermoid anal cancer: 13-year follow-up of the first randomised UKCCCR Anal Cancer Trial (ACT I). Br J Cancer. 2010 30;102(7):1123-8.

8. Bartelink H, Roelofsen F, Eschwege F, et al. Concomitant radiotherapy and chemotherapy is superior to radiotherapy alone in the treatment of locally advanced anal cancer: results of a phase III randomized trial of the European Organization for Research and Treatment of Cancer Radiotherapy and Gastrointestinal Cooperative Groups. J Clin Oncol. 1997;15(5):2040-9.

9. Flam M, John M, Pajak TF, et al. Role of mitomycin in combination with fluorouracil and radiotherapy, and of salvage chemoradiation in the definitive nonsurgical treatment of epidermoid carcinoma of the anal canal: results of a phase III randomized intergroup study. J Clin Oncol. 1996;14(9):2527-39.

10. Ajani JA, Winter KA, Gunderson LL, et al. Fluorouracil, mitomycin, and radiotherapy vs fluorouracil, cisplatin, and radiotherapy for carcinoma of the anal canal: a randomized controlled trial. JAMA.2008;299(16):1914-21.
11. Gunderson LL, Winter KA, Ajani JA, et al. Long-term update of US GI intergroup RTOG 98-11 phase III trial for anal carcinoma: survival, relapse, and colostomy failure with concurrent chemoradiation involving fluorouracil/ mitomycin versus fluorouracil/cisplatin. J Clin Oncol. 2012;30(35):4344-51.

12. James RD, Glynne-Jones R, Meadows $\mathrm{HM}$, et al. Mitomycin or cisplatin chemoradiation with or without maintenance chemotherapy for treatment of squamous-cell carcinoma of the anus (ACT II): a randomised, phase 3, openlabel, $2 \times 2$ factorial trial. Lancet Oncol.2013;14(6):516-24.

13. Glynne-Jones R, Sebag-Montefiore D, Meadows HM, et al. Best time to assess complete clinical response after chemoradiotherapy in squamous cell carcinoma of the anus (ACT II): a post-hoc analysis of randomised controlled phase 3 trial. Lancet Oncol. 2017;18(3):347-56.

14. Peiffert D, Tournier-Rangeard L, Gérard JP, et al. Induction chemotherapy and dose intensification of the radiation boost in locally advanced anal canal carcinoma: final analysis of the randomized UNICANCER ACCORD 03 trial. J Clin Oncol. 2012;30(16):1941-8.

15. Wegner RE, Abel S, Hasan S, et al. trends in radiation dose and technique for anal canal squamous cell carcinoma. Am J Clin Oncol. 2019;42(6):519-26.

16. Olsen JR, Moughan J, Myerson R, et al. Predictors of radiation therapy-related gastrointestinal toxicity from anal cancer dose-painted intensity modulated radiation therapy: secondary analysis of NRG Oncology RTOG 0529. Int J Radiat Oncol Biol Phys. 2017;98:400-8.

17. Olivatto LO, Vieira FM, Pereira BV, et al. Phase 1 study of cetuximab in combination with 5-fluorouracil, cisplatin, and radiotherapy in patients with locally 
advanced anal canal carcinoma. Cancer. 2013;119(16):2973-80.

18. Deutsch E, Lemanski C, Pignon JP, et al. Unexpected toxicity of cetuximab combined with conventional chemoradiotherapy in patients with locally advanced anal cancer: results of the UNICANCER ACCORD 16 phase II trial. Ann Oncol. 2013;24(11):2834-8.

19. Garg MK, Zhao F, Sparano JA, et al.Cetuximab Plus Chemoradiotherapy in Immunocompetent Patients With Anal Carcinoma: A Phase II Eastern Cooperative Oncology Group-American College of Radiology Imaging Network Cancer Research Group Trial (E3205). J Clin Oncol. 2017;35(7):718-26.

20. Casadei Gardini A, Passardi A, Fornaro L, et al. Treatment of squamous cell carcinoma of the anal canal: A new strategies with anti-EGFR therapy and immunotherapy. Crit Rev Oncol Hematol. 2018;123:52-56. 